\title{
Tomato Seeds Preferably Transmit Plant Beneficial Endophytes
}

\author{
Alessandro Bergna, ${ }^{1,2}$ Tomislav Cernava, ${ }^{2, \dagger}$ Manuela Rändler, ${ }^{2}$ Rita Grosch, ${ }^{3}$ Christin Zachow, ${ }^{1}$ and \\ Gabriele Berg ${ }^{2}$
}

${ }^{1}$ Austrian Centre of Industrial Biotechnology, Petersgasse 14, $8010 \mathrm{Graz}$, Austria; ${ }^{2}$ Institute of Environmental Biotechnology, Graz University of Technology, Petersgasse 12, 8010 Graz, Austria; and ${ }^{3}$ Department Plant-Microbe Systems, Leibniz Institute of Vegetable and Ornamental Crops, Theodor-Echtermeyer-Weg 1, 14979 Großbeeren, Germany

Accepted for publication 28 September 2018.

\section{ABSTRACT}

Endophytes with plant growth-promoting activity can improve the health and development of plants during all life stages. However, less is known about their stability and transmission across plant genotypes, habitats, and generations. By combining community and isolate analyses, we found that each plant habitat and genotype harbored distinct bacterial communities and plant growth-promoting bacteria (PGPB). Soil, root endosphere, and rhizosphere were the habitats with the highest bacterial diversity, while seeds hosted more selective communities. Seeds generated under field conditions showed traces of a bacterial community composition connected to the suppression of plant pathogens. In contrast, seeds of the successive generation grown in a pathogen-free and low-nutrient environment showed a predominance of bacteria that facilitate the uptake of nutrients. These modifications of the microbiome can be explained by an adaptation to prevalent environmental conditions. Cultivation approaches revealed microhabitat-specific PGPB that were assigned to various species of Bacillus, Stenotrophomonas, and Ralstonia. Tracking down these bacteria among the whole tomato plant allowed us to identify the seed as a primary vehicle of PGPB transmission. This previously undescribed vertical transmission of PGPB represents a strategy to maintain plant beneficial bacteria over generations and has an impact for the design of seed treatments.

Additional keywords: seed microbiota, Solanum lycopersicum.
The plant microbiota has been known to be one of the key determinants of plant health and productivity for more than a century (Philippot et al. 2013; Yan et al. 2017). It has the ability to contribute to multiple aspects in the functioning of the plant holobiont (Vandenkoornhuyse et al. 2015), such as (i) seed germination and growth support, (ii) nutrient supply, (iii) resistance against biotic stress factors (pathogen defense), (iv) resistance against abiotic factors, and (v) production of bioactive metabolites (Berg et al. 2015). Due to this importance, the factors that shaped the plant microbiota have been studied for a long time. Following a long debate, it is accepted that the plant genotype and soil quality are the crucial factors influencing the composition of the rhizosphere microbiota (Berg and

${ }^{\dagger}$ Corresponding author: Tomislav Cernava; E-mail: tomislav.cernava@tugraz.at

Funding: This project is part of the BestPass International Training Network funded by the European Union's Horizon 2020 research and innovation programme under the H20202 Marie Skłodowska-Curie Actions grant number 676480.

*The $\boldsymbol{e}$-Xtra logo stands for "electronic extra" and indicates that three supplementary figures and six supplementary tables are published online.

(C) 2018 The American Phytopathological Society
Smalla 2009). Both have an impact, but the extent depends on many factors (plant's morphology and secondary metabolism and soil type) and is triggered by plant root exudates and signaling (Badri and Vivanco 2009; Doornbos et al. 2012). The same question, namely whether the soil quality or the plant genotype determines the microbiota composition of the seeds, arose. For a long time, it was assumed that the emerging seedling is colonized by microorganisms from its surrounding environment, with soil being the main source, controlled by the plant through different strategies, such as the specific profile of root exudates and its immune system (SánchezCañizares et al. 2017; Shade et al. 2017; Truyens et al. 2014). Recently, an impact of the plant genotype on the microbial seed composition was identified (Adam et al. 2018; Rezki et al. 2018; Rybakova et al. 2017). This indicates that possibly both factors are involved, which was also used as a first hypothesis for this study. Moreover, there are many knowledge gaps; especially regarding the transmission and stability of the seed microbiota (Berg and Raaijmakers 2018). Vertical transmission would thus permit a "continuity of partnership" between the plant and its beneficial bacteria (Vannier et al. 2018), which leads us to our second hypothesis that the beneficial partners are transmitted from seed to seed. 
To evaluate our hypotheses, we have selected tomato as a model plant. Tomato (Solanum lycopersicum L.) is one of the most important vegetables; 177 million tons are harvested each year (FAOSTAT 2016). Tomato and other vegetables are an important part of a healthy diet, they can lower blood pressure, reduce risk of heart disease and stroke, prevent some types of cancer, lower risk of eye and digestive problems, and have a positive effect upon blood sugar (He et al. 2006). Although new findings recommend an increased intake of fruits and vegetables, the production and processing is associated with enormous losses up to $45 \%$ (FAO 2015). Soilborne pathogens, e.g., Fusarium, Rhizoctonia, and Verticillium, are one of the major factors that significantly limit crop yields (Oerke 2006). They are difficult to suppress, but recent advances in our understanding of the plant microbiota and of the mechanisms responsible for plant growth promotion and biological control of pathogens are opening the way to control them biologically (Mendes et al. 2011; Scherwinski et al. 2008). The microbiome of tomato has been widely characterized in its below ground compartment, mainly focusing on its capability to host beneficial bacterial endophytes conferring resistance to biotic and abiotic stresses (Liu et al. 2017; Upreti and Thomas 2015; Yan et al. 2003). What these approaches are missing, is the characterization of the microbiome of the aboveground plant parts, where the microbiota is less exposed to the effect of the soil (Ottesen et al. 2013) and thus the plant plays a stronger role in the selection of bacteria (Truyens et al. 2014), as well as a link to the seed microbiome.

The objective of this work was to unlock the tomato microbiome and understand (i) which are the main drivers of the microbiome composition, (ii) what is the impact of soil quality on the seed microbiome, and (iii) how the transmission of PGPB in the seed across generations works. The experimental design was based on greenhouse experiments and included samples analyzed from bulk soil, rhizosphere, root endosphere, seeds (first generation) as well as seeds harvested from the grown tomato plants at maturity (second generation) from two cultivars (Moneymaker and Hildares F1). An integrative approach based on amplicon sequencing and a bacterial strain collection was linked with bioinformatic analyses. It allowed the reconstruction of the tomato plant system's bacteriome and the location of beneficial bacteria among plant microhabitats.

\section{MATERIALS AND METHODS}

Experimental design. Microbiome-related analyses were performed using the tomato (Solanum lycopersicum) cultivars Moneymaker (Austrosaat AG, Austria) and Hildares F1 (Hild Samen $\mathrm{GmbH}$, Germany) grown under greenhouse conditions (approximately $24 / 20^{\circ} \mathrm{C}$ day/night temperature) at Graz Botanical Garden (Graz, Austria). Surface-sterilized seeds (first generation) were sown in pots (one seed per pot; 8 liters), filled with a mixture of sterile quartz-sand and diluvial sand (Rühlmann and Ruppel 2005) or commercial loamy soil (Ökohum GmbH, Herbertingen, Switzerland) in a proportion of 10:1. The substrate mixtures are considered as nutrient poor systems. Each tomato cultivar included 50 plants arranged in a randomized design. The seedlings were watered and fertilized once a week with a nutrient solution $(100 \mathrm{ml} / \mathrm{plant}$, the complete composition of the solution is reported in Supplementary Table S1) (Hoagland and Arnon 1950). For the analysis of the bacterial community in each quartz sand/soil mixture, eight pots without plants were watered and maintained under the same nutrient and greenhouse conditions as planted pots.

Sample collection and DNA isolation. Sampling of soil and plants was carried out 85 days after sowing (late flowering stage) followed by a second sampling at the ripening of fruits of Moneymaker plants (Supplementary Table S2). Soil samples were obtained from pots containing soil-sand mixture only. After removing the top layer $(2$ to $3 \mathrm{~cm}$ ) of soil with sterile tools, soil samples from the central section of each pot was collected and stored in sterile bags. Rhizospheric soil was obtained by shaking the root compartment and collecting the falling off material. Root and soil samples from each pot were stored in separated sterile polyethylene bags at $4^{\circ} \mathrm{C}$ until further processing on the following day. Tomato seeds from the second plant generation were extracted from the ripened fruits. Seeds of the second generation were harvested from 10 Moneymaker plants. For extracting the total microbial community DNA, both soil and rhizosphere material were initially suspended in $0.85 \%$ sodium chloride solution $(\mathrm{NaCl})$ and shaken for $30 \mathrm{~min}$. Root material was surface sterilized and processed according to the protocol described by Bragina et al. 2012). Briefly, $5 \mathrm{~g}$ of roots was washed, surface sterilized in a $3 \%$ sodium hypochlorite solution $(\mathrm{NaClO})$ for $5 \mathrm{~min}$ (manual shaking) and then again washed in sterile water three times. The plant material was imprinted on NA agar plates as a sterility check. Similarly, seeds of Moneymaker and Hildares F1 were washed in sterile water, divided in plastic vials ( 20 seeds per vial) with $4 \mathrm{ml}$ of $0.85 \% \mathrm{NaCl}$ and gently shaken for $4 \mathrm{~h}$. All plant samples (root and seed) were homogenized with mortar and pestle and suspended in $0.85 \% \mathrm{NaCl}$. Bacteria-containing pellets from both plant and soil specimens were collected by centrifugation $(20 \mathrm{~min}$ at $16.750 \mathrm{~g}$ ) and stored at $-70^{\circ} \mathrm{C}$.

DNA isolation, 16S rDNA PCR amplification, and sequencing. The aforementioned pellets were used for the total community DNA isolations. DNA was isolated with the FastDNA SPIN Kit for Soil and the FastPrep Instrument (MP Biomedicals, Santa Ana, CA) according to the manufacturer's protocol. Final aliquots of the total community DNA were further quantified and used as template for PCR amplifications (thermal cycler by Biometra GmbH, Jena, Germany) using Taq-\&GO Ready-to-use PCR Mix (MP Biomedicals) and the universal bacterial primer set 515f/ 806r (515f: 5'-GTGYCAGCMGCCGCGGTAA-3'; 806r: 5' GGACTACNVGGGTWTCTAAT-3') targeting the 16S rDNA hypervariable region 4 with the suggested PCR program $\left(94^{\circ} \mathrm{C}\right.$ for 3 min to denature the DNA, 35 cycles at $94^{\circ} \mathrm{C}$ for $45 \mathrm{~s}, 50^{\circ} \mathrm{C}$ for $60 \mathrm{~s}$, and $72^{\circ} \mathrm{C}$ for $90 \mathrm{~s} ; 10 \mathrm{~min}$ at $72^{\circ} \mathrm{C}$ for final extension) (Caporaso et al. 2011). Barcode sequences for multiplexing of the data were used as provided by the earth microbiome project (earthmicrobiome.org/). In addition, peptide nucleic acid PCR clamps were used to block the amplification of plastid and mitochondrial 16S rRNA gene sequences of plants during the PCR amplification. The amplicons were purified by using the Wizard SV Gel and PCR Clean-Up System (Promega, Madison, WI). A total of 86 barcoded samples were pooled equimolarly and sent for paired-end Illumina MiSeq sequencing (GATC Biotech, Germany) performed in two sequential batches. The $16 \mathrm{~S}$ rRNA Illumina libraries obtained from the sequencing company were deposited at the European Nucleotide Archive (ENA) under the project number PRJEB27033.

Data analysis of 16S rDNA amplicons for determination of microbial community structure. Acquired 16S rRNA gene sequences went through an initial quality check. Only forward reads were selected for further analyses due to substantially higher read quality when compared with reverse reads. Demultiplexing followed by quality filtering with QIIME (Quantitative Insights into Microbial Ecology, version 1.9.0; Caporaso et al. 2010) default parameters (Bokulich et al. 2013) was conducted for the whole dataset. High quality reads were dereplicated and clustered with a similarity threshold of $97 \%$ via VSEARCH (version 2.4.3). After creating a set of representative sequences, chimeras were filtered via both de novo reference-based approaches while mapping high quality sequences (vsearch) (Rognes et al. 2016). The taxonomical assignment was obtained by employing QIIME environment RDP 
(default parameters) in combination with the SILVA 16S database (release 128) (Pruesse et al. 2007). Unassigned operational taxonomic units (OTUs) and nonbacterial contaminants were filtered from the resulting OTU table. OTUs abundances have been rarefied via subsampling in QIIME environment to allow comparisons between samples. A consensus-table was obtained by averaging the subsampled tables. The description of the bacterial community structure was performed using a QIIME summarized table at phylum and family levels with samples belonging to the same microhabitat merged together. Graphical rendering of the community structure at phylum level was done with the opensource software Circos (Krzywinski et al. 2009) (Fig. 1). A more detailed description at family level was rendered with bar charts; the employment of analysis of variance (ANOVA) with false discovery rate correction allowed to identify bacterial families with significantly different abundances among tested sample groups. Alpha diversity was calculated and rendered at OTU level in the R environment with the Phyloseq package (McMurdie and Holmes 2013) using observed species, Chao 1, Shannon, and inverse Simpson measures. The PCoA plot was also generated with Phyloseq on an OTU table summarized at family level in QIIME. Selected OTUs were studied at more resolved taxonomic levels with the online nucleotide BLAST tool (https://blast.ncbi.nlm.nih. gov/).

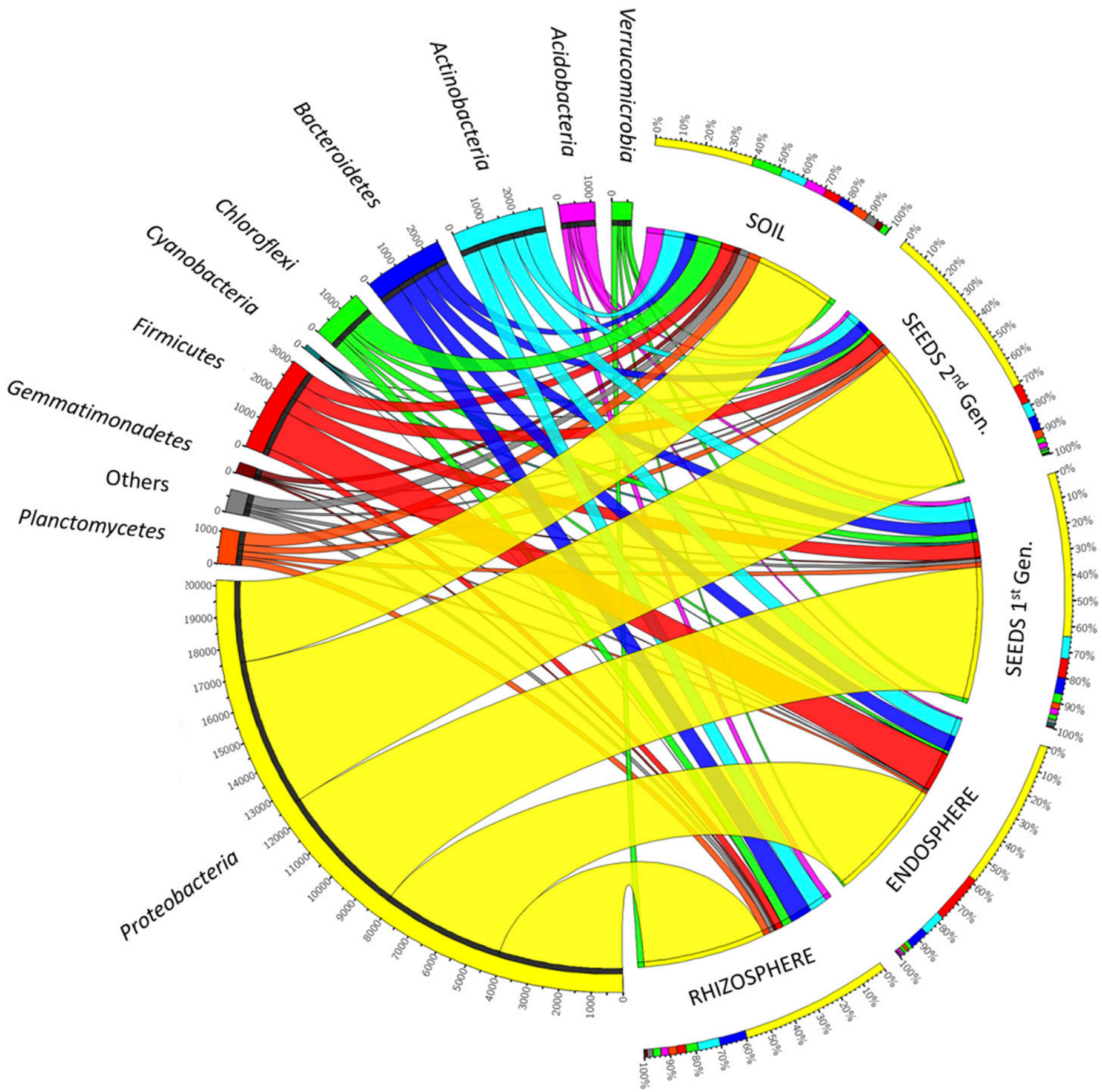

Fig. 1. Circular representation of the bacterial community structure (at phylum level) in different microhabitats associated with the tomato plant. Taxa with a proportion lower than $1 \%$ in all habitats are summarized as 'Others'. Values in the inner circle indicate the normalized number of reads assigned to the respective phylum. The visualization was rendered using the open-source software Circos (Krzywinski et al. 2009). 
Bacterial community structure and plant beneficial bacteria. To analyze and visualize how OTUs are partitioned between microhabitat an OTU network was generated in QIIME and subsequently rendered with the software Cytoscape v. 3.5.1 (Shannon et al. 2003). The network was constructed using an OTU table rarefied at 7,000 reads per sample after removal of singletons and doubletons. In order to increase the representativeness of the subset table, random subsampling was repeated 10 times and average values have been considered. In the resulting network, lines connect OTUs (circles) to the microhabitats they are related to. Each OTU circle was rendered with a radius correlated to the OTU abundance among all studied samples. OTUs with taxonomical assignments matching with the characterized isolates were as well subjected to manual BLAST searches (representative sequences) in order to identify overlaps (using the same algorithm and parameters). OTUs were represented with a circle proportional to the abundance and those found to be beneficial in the conducted assays were noted with their respective taxonomical identification.

Isolation and characterization of bacteria with plant growthpromoting traits. Bacterial strains were isolated from the tomato plant system (soil, rhizosphere, root endosphere, and seeds of both generations) using the protocol published by Bragina et al. (2012). The obtained homogeneous sample suspensions were plated on $10 \%$ tryptic soy agar $(\mathrm{pH}=7.3 \pm 0.2$, Sigma-Aldrich; Taufkirchen, Germany), water agar $(\mathrm{pH}=6.8 \pm 0.2)$, and $\mathrm{R} 2 \mathrm{~A}(\mathrm{pH}=7.2 \pm 0.2$, Sifin; Berlin, Germany). The selection of bacterial endophytes was carried out with a systematically randomized approach: solid media plates were divided in six equal parts and colonies of one of the six parts were collected, purified, and preserved. The ability of isolated strains to produce metabolites that, in other strains, have been associated with plant growth promoting abilities was assessed by the production of indol-3-acetic acid, siderophores as described in Berg et al. (2002), ACC-deaminase (Dworkin and Foster 1958), and acetoin (Murray and Baron 2007). Their ability to inhibit the growth of plant-pathogenic fungi was indirectly assessed based on chitinase production (Nagpure and Gupta 2013), and in dual-culture experiments using the pathogenic Fusarium oxysporum Fol 007 strain (Berg et al. 2002). In addition to classic antagonism tests, isolates were screened for the emission of growth-inhibiting bioactive volatile compounds (Cernava et al. 2015) with F. oxysporum Fol

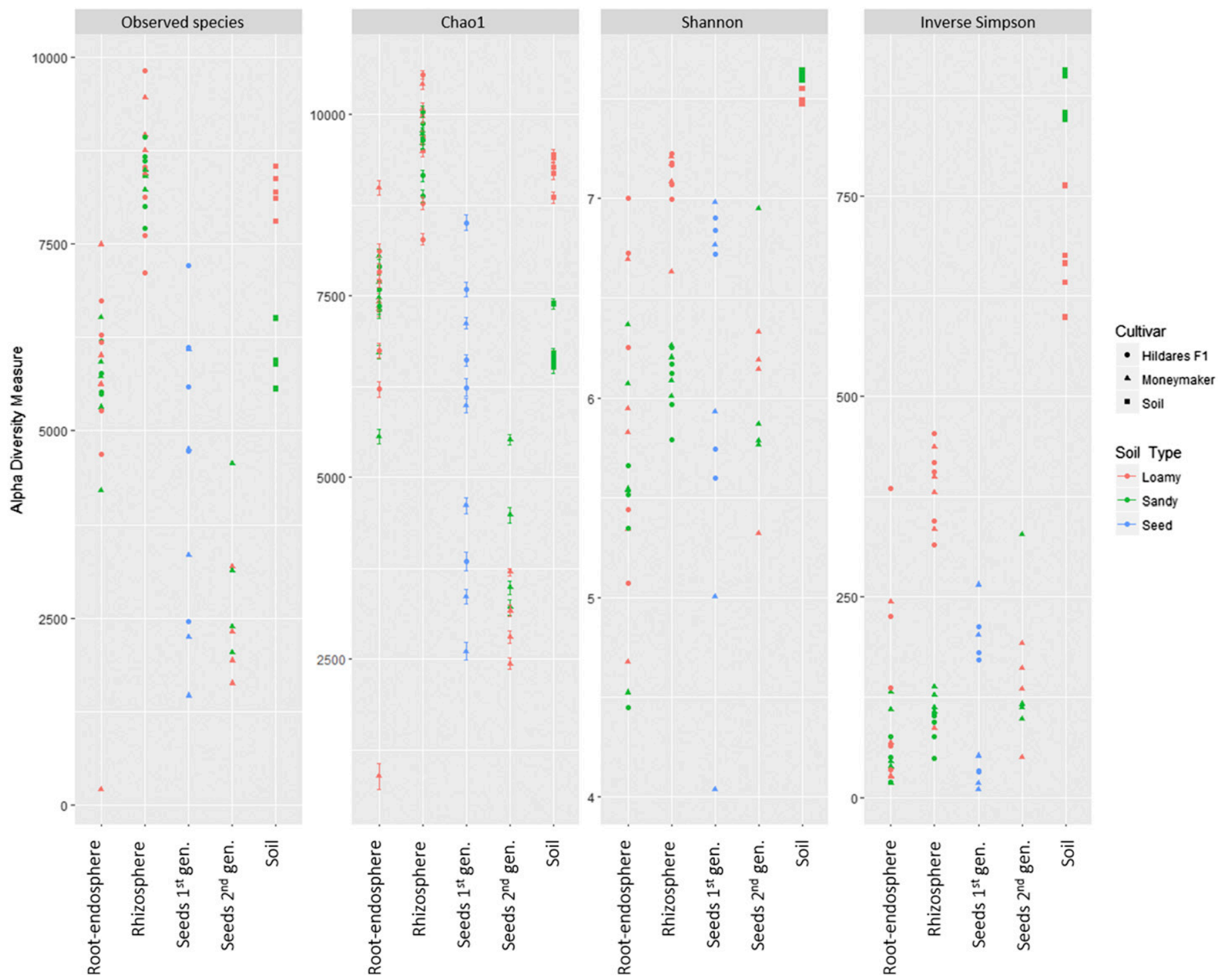

Fig. 2. Diversity assessment across all included samples and four different diversity measures: observed Species, Chao1, Shannon, and inverse Simpson. The combination of measures sensitive to rare operational taxonomic units (OTUs) (observed species and Chao1) and to dominant OTUs (inverse Simpson's index) provides a comprehensive assessment of bacterial diversity in the plant system. Samples are colored according to the soil type employed while the shape refers to the respective tomato cultivar. 
007 as model pathogen. Moreover, salt/drought tolerance and phosphate-solubilization capacity were assessed in additional experiments (Naik et al. 2008; Pikovskaya 1948) together with in planta assays for plant growth promotion (climate chamber conditions: temperature $($ day/night $)=25 / 20^{\circ} \mathrm{C}$, photoperiod: $16 \mathrm{~h}$, light intensity: $400 \mu \mathrm{mol} \mathrm{m} \mathrm{m}^{-2} \mathrm{~s}^{-1}$, humidity $70 \%$ ). Isolates with plant growth-promoting traits and other beneficial functions underwent taxonomical identification by $16 \mathrm{~S}$ rRNA gene sequencing and manual BLAST searches (https://blast.ncbi.nlm.nih.gov/) (Supplementary Table S3).

\section{RESULTS}

General structure of tomato-associated bacterial communities and diversity analyses. The DNA sequencing of marker genes from bacterial communities of two tomato cultivars (Moneymaker and Hildares F1) grown in two mixtures of quartz sand with two different soils (diluvial sand and loamy soil) resulted in a total of $32,411,312$ high quality reads. After discarding chimeras, singletons, chloroplast and non-bacterial reads, 21,369,607 reads remained with an average abundance of 318,949 reads per sample and a standard deviation of 276,419 reads. The bioinformatic reconstruction of the bacterial community identified a total of 13,928 distinct OTUs.

Proteobacteria were predominant in all microhabitats (Fig. 1). In the first-generation seeds, second-generation seeds, root endosphere, and rhizosphere $(64,71,69$, and $60 \%$, respectively) this phylum covered more than half of the bacterial community while in soil it was less representative (40\%). Other representative phyla were Firmicutes (up to $19 \%$ in the endosphere), Actinobacteria, and Bacteroidetes. All three were equally distributed among microhabitats, averaging 9, 8, and 7\%, respectively. Chloroflexi,
Acidobacteria, and Planctomycetes were mostly found in soil samples (respectively 12, 8, and 6\% in soil). Among all plant compartments (seeds of both first and second generation and root endosphere), Pseudomonadaceae and Comamonadaceae were the most occurring bacterial families. Considering both cultivars, the seeds of the first generation were characterized by a selective bacterial community, where only few taxa were dominant: Burkholderiaceae (19\%), Pseudomonadaceae (7\%), and Comamonadaceae (6\%). Conversely, the soil was characterized by the high abundance of Anaerolineaceae (6\%) and Planctomycetaceae (5\%). In the rhizosphere, Comamonadaceae (8\%), Pseudomonadaceae (7\%), and Flavobacteriaceae (5\%) represented the most abundant families, while the root endosphere was dominated by the family Pseudomonadaceae (18\%) followed by Comamonadaceae (6\%), Bacillaceae (6\%), and Rhizobiaceae (6\%). Even if decreased, the family Burkholderiaceae was assigned almost exclusively to seed specimens (19\% in the first-generation seeds and $3 \%$ in the secondgeneration seeds).

The diversity among microhabitats was evaluated with alpha diversity metrics (Fig. 2). To better describe the bacterial community composition, we selected four different measures sensitive to rare OTUs (observed species and Chao1), to dominant OTUs (inverse Simpson's index) and incorporating both richness and evenness (Shannon). Values for observed species varied between 7,505 and 13,725, while Chao1 values between 8,662 and 13,785 were observed and inverse Simpson indices between 72 and 1,070 (Supplementary Table S4). Soil, rhizosphere, and root endosphere were the microhabitats with the highest diversity while seeds hosted more selective communities in both generations. Soil diversity varied among loamy and sandy soil: the loamy soil was characterized by a higher number of rare OTUs and lower of dominant OTUs when compared with sandy soil. Conversely,

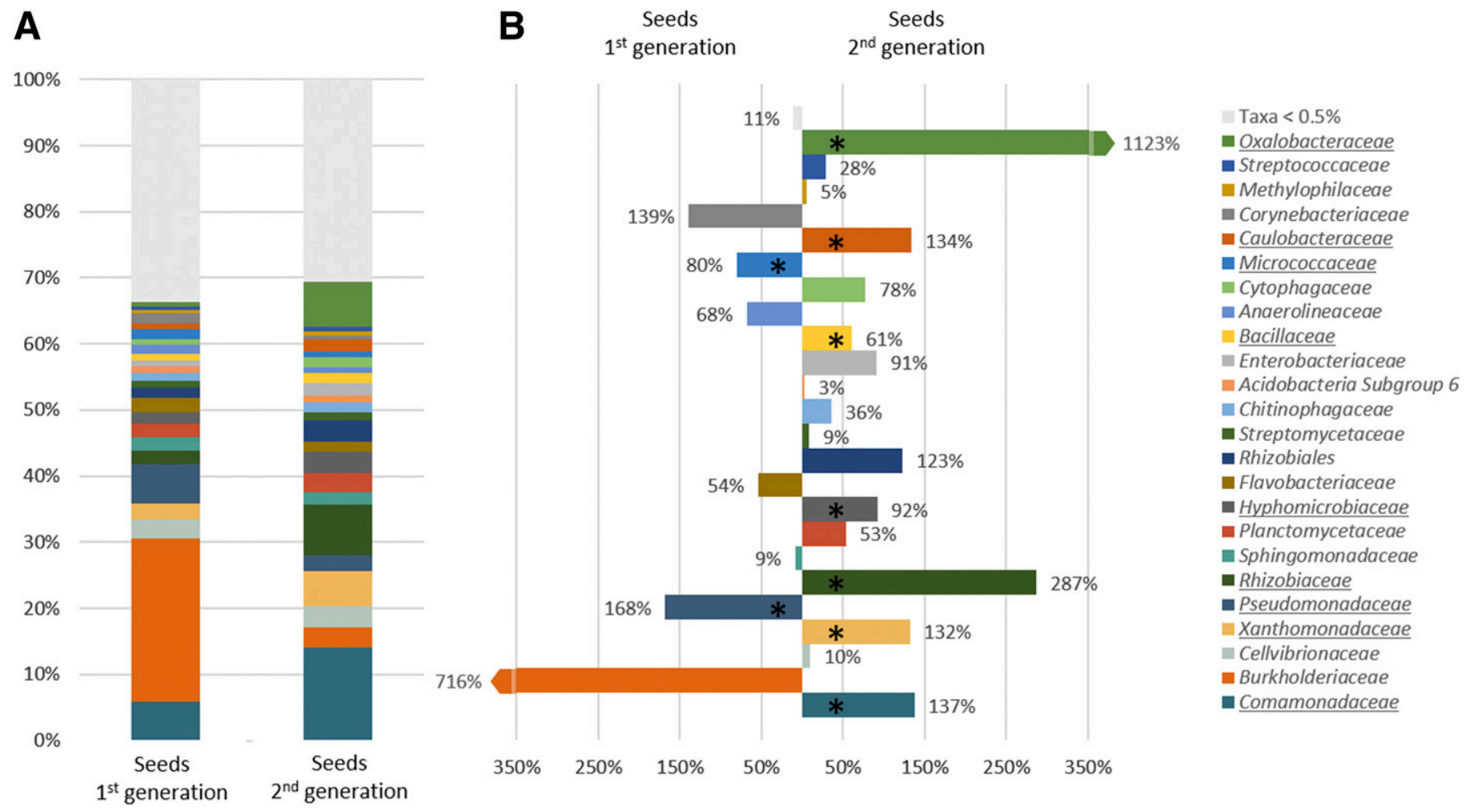

Fig. 3. Bacterial community composition of the tomato seeds across two generations. A, Bar charts represent the composition of the bacterial community (only key taxa were included, $>0.5 \%$ ) among two generations: seeds employed for generating the plants (left) and harvested seeds (right). B, Horizontal plots represent the relative variation of key taxa among generation types (statistically significant differences are marked with an asterisk ( ${ }^{\star}$ ) and corresponding taxa names are underlined). 
rhizosphere and root endosphere hosted a comparable number of rare OTUs, but a lower number of dominant OTUs in plants grown on sandy soil.

Bacterial community structure of tomato seeds across generations. After harvesting root tissues and soil, a total of 10 Moneymaker plants were left in the nonacclimated greenhouse environment to harvest their seeds upon ripening. The comparison between all bacterial communities analyzed saw a similar pattern in key taxa composition (Fig. 3A). Significant differences were attributable to taxa that dominated the community of this cultivar: the family Burkholderiaceae dominated the first generation (25\%) and drastically decreased in the second generation $(3 \%)$ with Pseudomonadaceae also decreasing at the same time (from 6 to $2 \%$ ). Conversely, families Comamonadaceae (14\%), Rhizobiaceae (8\%), and Oxalobacteraceae (7\%) dominated the bacterial community of the second generation after a substantial increment over the studied generation span (these taxa represented, respectively, 6, 2 , and $0.5 \%$ of the bacterial community of the first generation) (Fig. $3 \mathrm{~B})$. The variation in the abundance of nine bacterial families was found to be significant by ANOVA after false discovery rate correction. Manual BLAST of the 10 single OTUs assigned to the family Burkholderiaceae resulted in the identification of 19 potential taxonomical assignments at species level (best-hit results are shown in Supplementary Table S5). Additionally, the OTU composition of the family Oxalobacteraceae was similarly investigated by manual BLAST of representative sequences and revealed Massilia sp. to be the most frequent assignment.

Cultivar-driven variations in the bacterial communities of tomato microhabitats. Different plant compartments hosted distinctive bacterial communities depending on the cultivar or soil employed. Among different seed cultivars, even if characterized by a comparable diversity, prevailing bacteria (abundance $>0.5 \%$ in each considered microhabitat) were demarked by a higher presence of taxa belonging to Rhodobacteraceae in Hildares F1 seeds (from 0.5 to $1 \%$ ) and of Cyanobacteria ML635J-21, Staphylococcaceae, and Burkholderiaceae in Moneymaker first-generation seeds (Supplementary Fig. S1). In a similar way, root endosphere bacterial communities showed an increment of Streptomycetaceae, Bacillaceae, Cellvibrionaceae in Hildares F1, and of Xanthomonadaceae, Rhizobiales, and Nocardioidaceae in Moneymaker (Supplementary Fig. S2). PCoA plotting of the beta diversity (pairwise sample dissimilarity; Fig. 4) showed dissimilarities among analyzed samples. Major variations were visible among the two generations of Moneymaker seeds: while in the first generation

PCoA on Bray Curtis distance, Soil type driven modifications

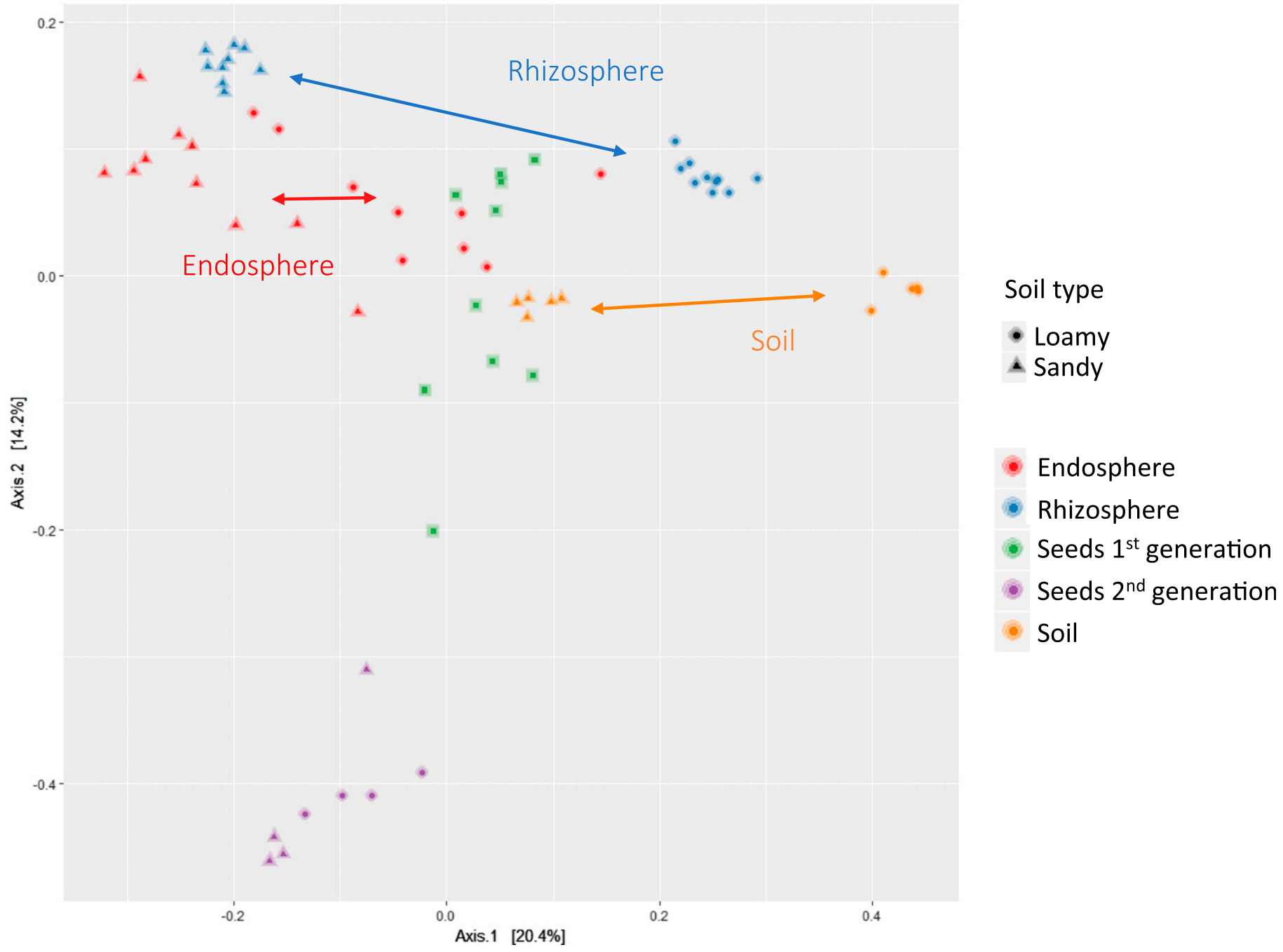

Fig. 4. PCoA plot with distances among samples based on the bacterial community composition. Samples are colored according to the different microhabitats. The soil types that were used to grow the tomato plants are denoted with different shapes. Seed bacteriome analyses with the second plant generation were only conducted with tomato cultivar Moneymaker. 
the community resulted similar to other plant compartments (adjacent in the plot), the shift induced in the second generation showed a substantial modification in the community. Differences among soil sources were also visible in the plot: loamy and sandy bulk soils clearly hosted different communities as rhizosphere and, to a lower extent, root endosphere (Supplementary Fig. S3).

Tracking down beneficial bacteria inside plant compartments and across seed generations. To understand which PGPB are distributed across the plant system, an OTU network was created by graphically linking OTUs to the corresponding habitat (Fig. 5). With the organization of OTUs in compartments-specific clusters, the abundance and taxonomical diversity of shared and exclusive OTUs was assessed.

The bacterial community of soil was characterized by the highest taxonomical diversity and number of OTUs exclusive for a single compartment. Accounting the whole plant system, OTUs attributed to Acidobacteria were present almost exclusively in soil and rhizosphere-connected clusters but with a detectable presence in seeds. OTUs assigned to Proteobacteria composed the majority in clusters linked to plant compartments. Firmicutes OTUs were specifically allocated in root endosphere as peculiar for this habitat or as shared with the soil.

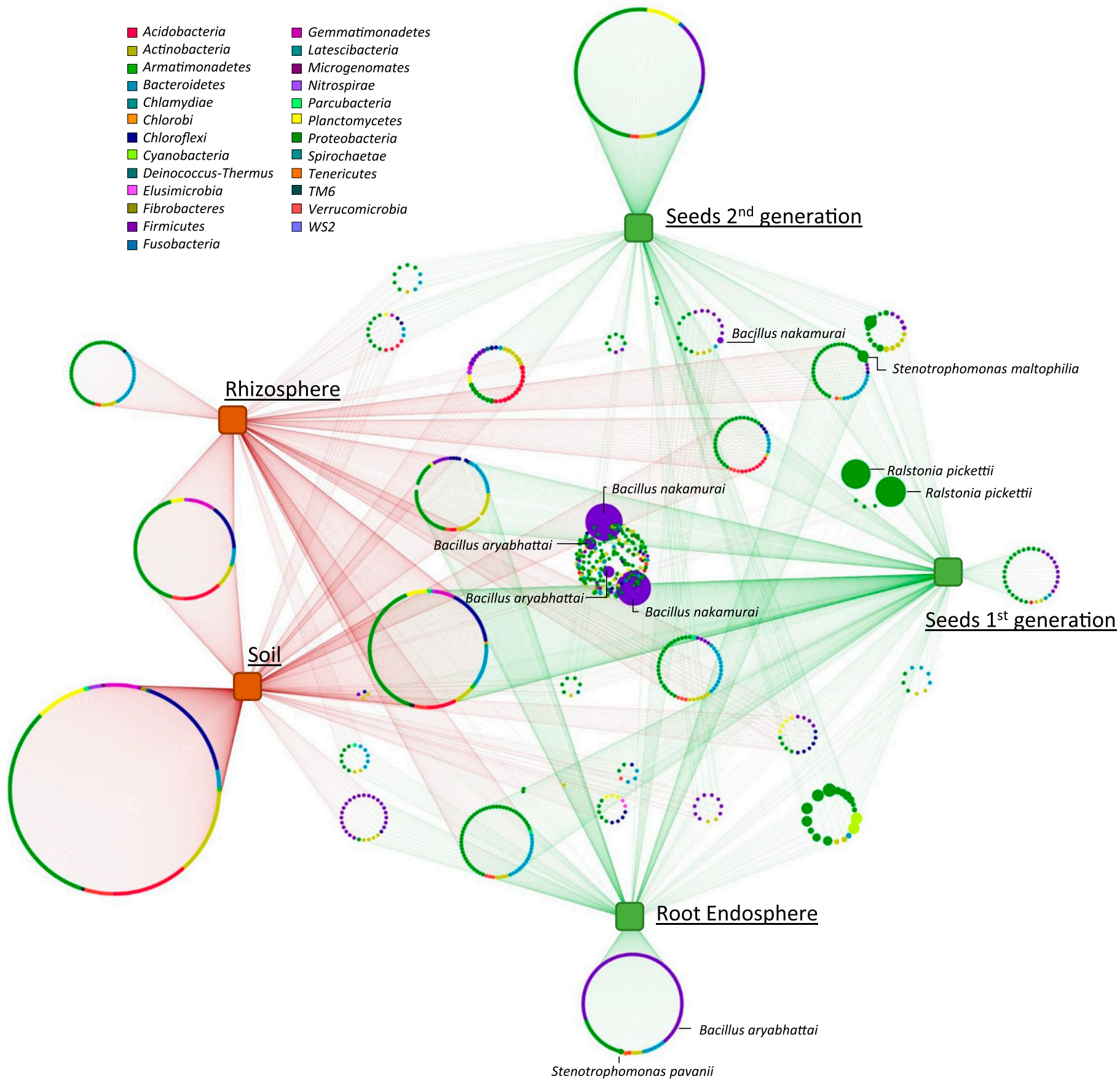

Fig. 5. Operational taxonomic unit (OTU) network of the tomato plant bacterial community structure. Dots represent single OTUs while labeled squares represent samples. Edges connecting samples with OTUs are colored to discern between soil (brown) and plant (green) habitats. Nodes are colored according to taxonomic assignments (phylum level). OTUs that correspond to isolated bacteria are highlighted with a node size proportional to their abundance. Labels highlight all plant growth-promoting bacteria in the network. 
A total number of 5,153 bacteria was isolated from the tomato plant system. Isolates were characterized and selected for PGP properties and antagonistic effects against $F$. oxysporum. Following the screening, 19 isolates showed multiple positive traits and were thus considered for further analyses (Supplementary Table S6). Most of these bacterial strains showed the ability to produce siderophores, ACC-deaminase, and salt/drought tolerance. Moreover, in vivo tests showed the ability of Bacillus aryabhattai C6b and Microbacterium flavescens $\mathrm{C} 7$ to promote plant growth in tomato plant in absence of stressors.

Five of these beneficial phenotypes were also found in the amplicon data reconstruction of the tomato plant bacterial community by $16 \mathrm{~S}$ rRNA gene comparison (Bacillus aryabhattai, Bacillus nakamurai, Ralstonia pickettii, Stenotrophomonas maltophilia, and Stenotrophomonas pavanii). OTUs belonging to these five plant beneficial taxa have been highlighted in the network analysis (Fig. 5). Both members of Bacillus spp. were found, in the core of the studied plant microbial communities with a single instance of OTU assigned to Bacillus nakamurai being shared among the root endosphere and second-generation seeds. Ralstonia pickettii was in both soil and first-generation seeds and has been found in the endosphere and in the seeds of the second generation (Fig. 6). While Stenotrophomonas pavanii was detected only in the root endosphere, Stenotrophomonas maltophilia was present in the seeds of the first generation and colonized the endosphere, the seeds of the second generation, and the rhizosphere.

\section{DISCUSSION}

Our results showed that soil bacterial community composition has a high impact on the bacterial community of below ground compartments (rhizosphere, root endosphere) in both tomato cultivars. However, the effect is progressively lowering from the rhizosphere to the root endosphere and finally to the seeds. At the same time, the seed bacterial community analysis across a generation identified a continuous turnover of the seed bacterial assemblage that might be triggered by environmental conditions. Interestingly, tracking down beneficial bacteria inside the whole plant system allowed us to identify plant seeds as a primary vehicle for transmission of PGPB.

Habitat specificity and key taxa in the tomato plant bacteriome. Plant seeds constitute the basis for each new plant generation and thus are essential determinants for the plant's early development. In the present study we found that the bacterial community of each plant habitat showed distinct fingerprints and harbored different PGPB. However, we found high cross-habitat similarity at phylum level; Proteobacteria were the most representative phylum within the whole plant system confirming previous findings (Bulgarelli et al. 2013). Soil, rhizosphere, and root endosphere were the microhabitats with the highest diversity, while seeds hosted more selective communities in both generations. As example, Acidobacteria were found to be under the detection threshold in the root endosphere as already described in a related
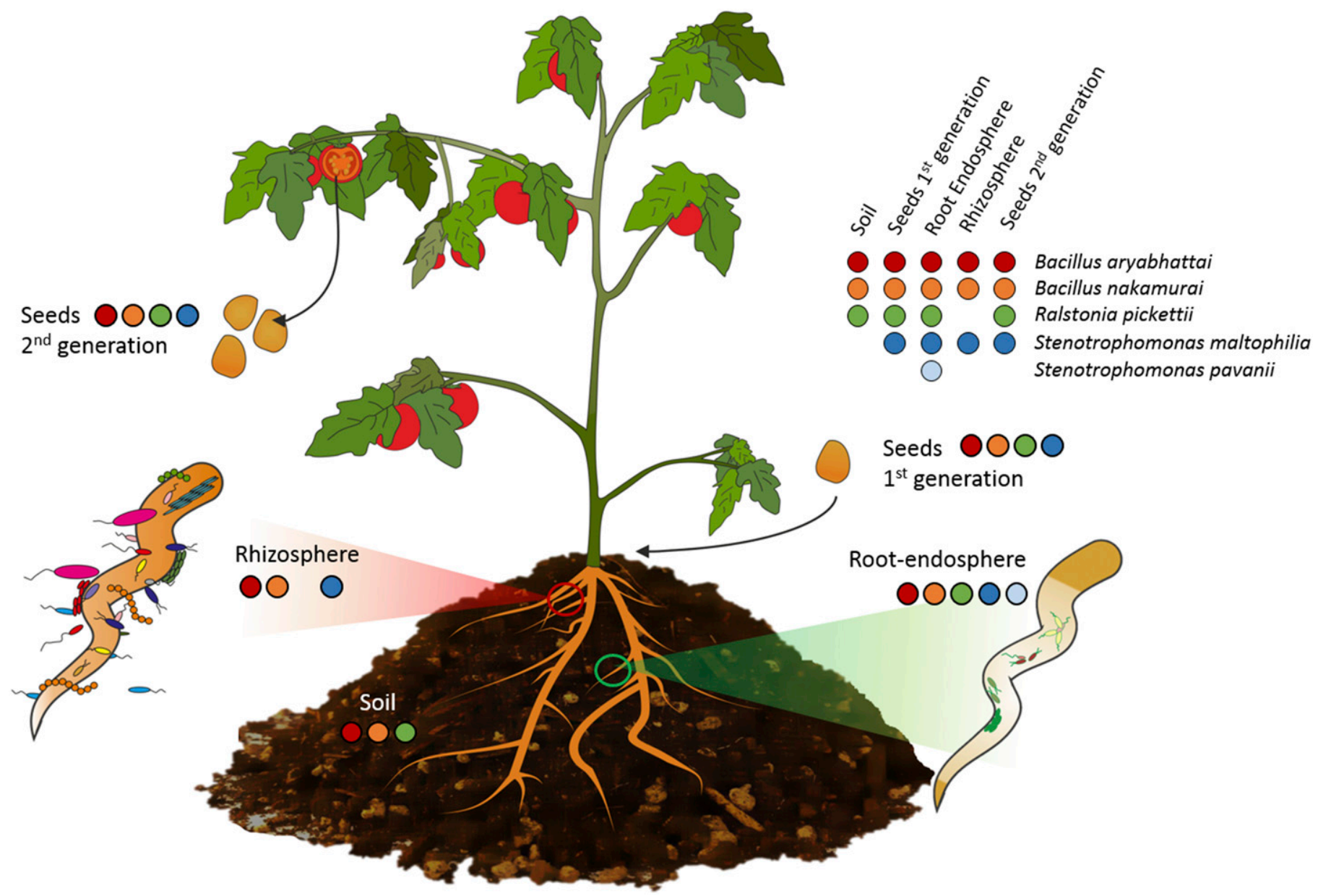

Fig. 6. Graphical representation of the plant system including microhabitat associations of analyzed isolates from the tomato endosphere. All identified bacteria with plant growth-promoting effects were linked to the microhabitats they were found to be associated with by colored dots. Both seed generations are included in the model. 
study (Santoyo et al. 2016); however, seeds of both generations have been found enriched with members of this phylum, thus the plant endosphere creates a bridge between soil and the seeds. Members of this phylum have been recently described as possessing growth-promoting effects associated to auxin production (Kielak et al. 2016) and are therefore connected to the regulation of seed germination, increment in roots and shoots biomass as well as morphological changes in root system (Shu et al. 2016). Although being an aboveground-located structure in tomato plants, the seed is a quiescent structure designed to be biochemically active only when in contact with the soil. For this reason, it is in the interest of the plant to allocate and enrich this compartment with soil-compatible bacteria using the rhizosphere and the root endosphere as a roadway inside the plant.

Plant genotype and soil bacterial community-both factors shaped the seed bacteriome. In the rhizosphere we found a strong impact of the soil microbiota comparable to other studies (De Ridder-Duine et al. 2005; Inceoğlu et al. 2012), and even if less evident, this signature was still visible in root endosphere. Interestingly, we found no instance of this phenomenon in the bacterial community of second-generation seeds grown in different soil types. Although the underlying mechanisms are currently unknown, the seed microbiota could be a feature of below ground plant compartments that is specifically shaped under influence of the plant. The sequencing data shows that seeds of the first and second generation hosted a different bacterial community with the latter also diverging from the other studied plant microhabitats. Interestingly, the difference among seeds was not ascribable to a completely different structure of the bacterial community but to the modification of dominant taxa belonging to Burkholderiaceae in the first generation and Oxalobacteraceae in the second generation. Members of Burkholderiaceae are known in biological control primarily for the outstanding ability to produce various antifungal compounds (Eberl and Vandamme 2016). Ralstonia piketii, that was found preeminent among Burkholderiaceae, represents a niche-competing microorganism that could occupy the ecological niche of pathogenic Ralstonia spp. For these reasons, the consistent decrement over a generation of this bacterial species when passing from field condition (seed producer) to a pathogen-free environment (controlled greenhouse) could be indicative for the connection of the seed bacterial assemblage to environmental conditions. In the same way, we found evidence of environment-driven modifications in the seed microbiome of the second generation. Massilia (Oxalobacteraceae) has already been described as exhibiting attributes related to plant growth in vitro (Hrynkiewicz et al. 2010; Weinert et al. 2010) and as highly variating across medium modification stages (Ofek et al. 2012). Both these findings support our findings related to the plant's transitioning from a nutrient rich substrate (field) to a nutrient-poor substrate ( $90 \%$ sand). Similarly, also the plant-beneficial bacterium Stenotrophomonas maltophilia was found to be increased in the second generation of the seed. Bacteria belonging to Stenotrophomonas spp. have been extensively used in agriculture as biocontrol agents for their ability to promote plant growth and produce antimicrobial compounds that protect plants (Ryan et al. 2009), therefore also confirming our hypothesis.

The distribution of PGPB across the tomato plant system. Our reconstruction of the bacterial community included tracing of beneficial bacteria that were isolated and characterized from tomato plants. We propose a model (Fig. 6) where, despite the large bacterial diversity harbored by the soil, the seed represents a major vehicle of transmission for PGPB. This finding introduces the idea that new isolation strategies for plant-growth beneficial bacteria should focus less on the high biodiversity of the soil and more on the plant with emphasis on the seed. Most of the microorganisms within seeds are in a dormant stage, therefore it is difficult to isolate them in a traditional procedure. However, isolation after a certain period after germination and growth under gnotobiotic conditions is more promising (Adam et al. 2018) and allows the exploit of this important bioresource for novel seed treatment strategies.

Cross-kingdom similarities can be suggested for vertical microbiota transmission. Mendes and Raaijmakers (2015) already described cross-kingdom similarities in microbiota functions. In the present study, we found certain parallels between the plant's seed microbiome and the human placenta microbiome. Aagaard et al. (2014) described the placenta's microbiome as unique in the human body, with the next closest microhabitat with a similar composition being the mouth cavity. Both microbiotas harbor a unique microbial community that substantially differs from that of spatially close microhabitats. It was also shown that mammals equip their offspring with beneficial microorganisms during birth (Blaser 2006). Likewise, plants can transfer a selected community to the next generation over their seeds or vegetative organs (Vannier et al. 2018). A similar phenomenon was also observed for mosses as well as lichens, which equip their propagules with a beneficial, bacterial community (Aschenbrenner et al. 2014; Bragina et al. 2012). This vertical transmission allows "continuity of partnership" between the plant and its symbionts within the holobiont concept (Vandenkoornhuyse et al. 2015; Vannier et al. 2018).

With this study, we intended to understand how PGPB are distributed across the plant system while studying how they are influenced by plant genotype and soil quality. We reconstructed the microbiome of the tomato plant considering four key microenvironments: the soil, the seeds, the rhizosphere, the root endosphere, and the seeds harvested at plant maturity. Our results showed that the plant genotype has no substantial influence on the bacterial community of below ground plant compartments that are instead tightly connected to soil quality. Deepening studies of the microbial composition of seeds across generations provided instances of how this compartment represents an important vehicle for the transmission of PGPB. The overall findings suggest that plant seeds have a key role as carriers of PGPB and are hotspots for their isolation. Detailed mechanisms on how environmental conditions can influence the selection process for seed endophytes and adjust the bacterial assemblage to a new, potentially advantageous composition remain to be explored.

\section{ACKNOWLEDGMENTS}

We thank C. Berg and his team of the Graz Botanical Garden (Graz, Austria) for skillful assistance in the greenhouse experiments.

\section{LITERATURE CITED}

Aagaard, K., Ma, J., Antony, K. M., Ganu, R., Petrosino, J., and Versalovic, J. 2014. The placenta harbors a unique microbiome. Sci. Transl. Med. 6:237.

Adam, E., Bernhart, M., Müller, H., Winkler, J., and Berg, G. 2018. The Cucurbita pepo seed microbiome: Genotype-specific composition and implications for breeding. Plant Soil 422:35-49.

Aschenbrenner, I. A., Cardinale, M., Berg, G., and Grube, M. 2014. Microbial cargo: Do bacteria on symbiotic propagules reinforce the microbiome of lichens? Environ. Microbiol. 16:3743-3752.

Badri, D. V., and Vivanco, J. M. 2009. Regulation and function of root exudates. Plant Cell Environ. 32:666-681.

Berg, G., and Raaijmakers, J. M. 2018. Saving seed microbiomes. ISME J. 12: 1167-1170.

Berg, G., Roskot, N., Steidle, A., Eberl, L., Zock, A., and Smalla, K. 2002. Plantdependent genotypic and phenotypic diversity of antagonistic rhizobacteria 
isolated from different Verticillium host plants. Appl. Environ. Microbiol. 68:3328-3338

Berg, G., Rybakova, D., Grube, M., and Koeberl, M. 2015. The plant microbiome explored: Implications for experimental botany. J. Exp. Bot. 67: 995-1002.

Berg, G., and Smalla, K. 2009. Plant species and soil type cooperatively shape the structure and function of microbial communities in the rhizosphere. FEMS Microbiol. Ecol. 68:1-13.

Blaser, M. J. 2006. Who are we? EMBO Reports Crit. 7.

Bokulich, N. A., Subramanian, S., Faith, J. J., Gevers, D., Gordon, I., Knight, R., Mills, D. A., and Caporaso, J. G. 2013. Quality-filtering vastly improves diversity estimates from Illumina amplicon sequencing. Nat. Methods 10:57-59.

Bragina, A., Berg, C., Cardinale, M., Shcherbakov, A., Chebotar, V., and Berg, G. 2012. Sphagnum mosses harbour highly specific bacterial diversity during their whole lifecycle. ISME J. 6:802-813.

Bulgarelli, D., Schlaeppi, K., Spaepen, S., van Themaat, E. V. L., and SchulzeLefert, P. 2013. Structure and functions of the bacterial microbiota of plants. Annu. Rev. Plant Biol. 64:807-838.

Caporaso, J. G., Kuczynski, J., Stombaugh, J., Bittinger, K., Bushman, F. D., Costello, E. K., Fierer, N., Peña, A. G., Goodrich, J. K., Gordon, J. I., Huttley, G. A., Kelley, S. T., Knights, D., Koenig, J. E., Ley, R. E., Lozupone, C. A., McDonald, D., Muegge, B. D., Pirrung, M., Reeder, J., Sevinsky, J. R., Turnbaugh, P. J., Walters, W. A., Widmann, J., Yatsunenko, T., Zaneveld, J., and Knight, R. 2010. QIIME allows analysis of high-throughput community sequencing data. Nat. Methods 7:335-336.

Caporaso, J. G., Lauber, C. L., Walters, W. A., Berg-Lyons, D., Lozupone, C. A., Turnbaugh, P. J., Fierer, N., and Knight, R. 2011. Global patterns of 16S rRNA diversity at a depth of millions of sequences per sample. Proc. Natl. Acad. Sci. 108:4516-4522.

Cernava, T., Aschenbrenner, I. A., Grube, M., Liebminger, S., and Berg, G. 2015. A novel assay for the detection of bioactive volatiles evaluated by screening of lichen-associated bacteria. Front. Microbiol. 6:1-9.

De Ridder-Duine, A. S., Kowalchuk, G. A., Klein Gunnewiek, P. J. A., Smant, W., Van Veen, J. A., and De Boer, W. 2005. Rhizosphere bacterial community composition in natural stands of Carex arenaria (sand sedge) is determined by bulk soil community composition. Soil Biol. Biochem. 37: 349-357.

Doornbos, R. F., Van Loon, L. C., and Bakker, P. A. H. M. 2012. Impact of root exudates and plant defense signaling on bacterial communities in the rhizosphere. A review. Agron. Sustain. Dev. 32:227-243.

Dworkin, M., and Foster, J. W. 1958. Experiments with some microorganisms which utilize ethane and hydrogen. J. Bacteriol. 75:592-603.

Eberl, L., and Vandamme, P. 2016. Members of the genus Burkholderia: Good and bad guys. F1000 Res. 5:1007.

FAO. 2015. Food losses and waste. http://www.fao.org/food-loss-and-foodwaste/en/ (Accessed 18 December 2018)

FAOSTAT. 2016. Food and Agriculture Organization of the United Nations FAOSTAT database. http://www.fao.org/faostat/en/ (Accessed 18 December 2018)

He, F. J., Nowson, C. A., and MacGregor, G. A. 2006. Fruit and vegetable consumption and stroke: Meta-analysis of cohort studies. Lancet 367: 320-326.

Hoagland, D. R., and Arnon, D. I. 1950. The water-culture method for growing plants without soil. Calif. Agric. Exp. Stn. Circ. 347:1-32.

Hrynkiewicz, K., Baum, C., and Leinweber, P. 2010. Density, metabolic activity, and identity of cultivable rhizosphere bacteria on Salix viminalis in disturbed arable and landfill soils. J. Plant Nutr. Soil Sci. 173:747-756.

Inceoğlu, Ö., Salles, J. F., and van Elsas, J. D. 2012. Soil and cultivar type shape the bacterial community in the potato rhizosphere. Microbiol. Ecol. 63: 460-470.

Kielak, A. M., Cipriano, M. A. P., and Kuramae, E. E. 2016. Acidobacteria strains from subdivision 1 act as plant growth-promoting bacteria. Arch. Microbiol. 198:987-993.

Krzywinski, M., Schein, J., Birol, I., Connors, J., Gascoyne, R., Horsman, D., Jones, S. J., and Marra, M. A. 2009. Circos: an information aesthetic for comparative genomics. Genome Res. 19:1639-1645.

Liu, K., Newman, M., McInroy, J. A., Hu, C. H., and Kloepper, J. W. 2017. Selection and assessment of plant growth-promoting rhizobacteria for biological control of multiple plant diseases. Phytopathology 107:928-936.

McMurdie, P. J., and Holmes, S. 2013. Phyloseq: An R package for reproducible interactive analysis and graphics of microbiome census data. PLoS One 8: e61217.
Mendes, R., Kruijt, M., de Bruijn, I., Dekkers, E., van der Voort, M., Schneider, J. H., Piceno, Y. M., DeSantis, T. Z., Andersen, G. L., Bakker, P. A., and Raaijmakers, J. M. 2011. Deciphering the rhizosphere microbiome for disease-suppressive bacteria. Science 332: $1097-1100$

Mendes, R., and Raaijmakers, J. M. 2015. Cross-kingdom similarities in microbiome functions. ISME J. 9:1905-1907.

Murray, P. R., and Baron, E. J. 2007. Manual of Clinical Microbiology. 9th ed. ASM Press, Washington, D.C.

Nagpure, A., and Gupta, R. K. 2013. Purification and characterization of an extracellular chitinase from antagonistic Streptomyces violaceusniger. J. Basic Microbiol. 53:429-439.

Naik, P. R., Raman, G., Narayanan, K. B., and Sakthivel, N. 2008. Assessment of genetic and functional diversity of phosphate solubilizing fluorescent pseudomonads isolated from rhizospheric soil. BMC Microbiol. 8:1-14.

Oerke, E. 2006. Crop losses to pests. J. Agric. Sci. 144:31-43.

Ofek, M., Hadar, Y., and Minz, D. 2012. Ecology of root colonizing Massilia (Oxalobacteraceae). PLoS One 7:e40117.

Ottesen, A. R., González Peña, A., White, J. R., Pettengill, J. B., Li, C., Allard, S., Rideout, S., Allard, M., Hill, T., Evans, P., Strain, E., Musser, S., Knight, R., and Brown, E. 2013. Baseline survey of the anatomical microbial ecology of an important food plant: Solanum lycopersicum (tomato). BMC Microbiol. 13:114.

Philippot, L., Raaijmakers, J. M., Lemanceau, P., and Van Der Putten, W. H. 2013. Going back to the roots: The microbial ecology of the rhizosphere. Nat. Rev. Microbiol. 11:789-799.

Pikovskaya, R. I. 1948. Mobilization of phosphorus in soil in connection with vital activity of some microbial species. Microbiologiya 17:362-370.

Pruesse, E., Quast, C., Knittel, K., Fuchs, B. M., Ludwig, W., Peplies, J., and Glöckner, F. O. 2007. SILVA: A comprehensive online resource for quality checked and aligned ribosomal RNA sequence data compatible with ARB. Nucleic Acids Res. 35:7188-7196.

Rezki, S., Campion, C., Simoneau, P., Jacques, M. A., Shade, A., and Barret, M. 2018. Assembly of seed-associated microbial communities within and across successive plant generations. Plant Soil 422:67-79.

Rognes, T., Flouri, T., Nichols, B., Quince, C., and Mahé, F. 2016. VSEARCH: A versatile open source tool for metagenomics. PeerJ 4:e2584.

Rühlmann, J., and Ruppel, S. 2005. Effects of organic amendments on soil carbon content and microbial biomass-results of the long-term box plot experiment in Grossbeeren. Arch. Agron. Soil Sci. 51:163-170.

Ryan, R. P., Monchy, S., Cardinale, M., Taghavi, S., Crossman, L., Avison, M. B., Berg, G., van der Lelie, D., and Dow, J. M. 2009. The versatility and adaptation of bacteria from the genus Stenotrophomonas. Nat. Rev. Microbiol. 7:514-525.

Rybakova, D., Mancinelli, R., Wikström, M., Birch-Jensen, A. S., Postma, J., Ehlers, R. U., Goertz, S., and Berg, G. 2017. The structure of the Brassica napus seed microbiome is cultivar-dependent and affects the interactions of symbionts and pathogens. Microbiome 5:104.

Sánchez-Cañizares, C., Jorrín, B., Poole, P. S., and Tkacz, A. 2017. Understanding the holobiont: the interdependence of plants and their microbiome. Curr. Opin. Microbiol. 38:188-196.

Santoyo, G., Moreno-Hagelsieb, G., del Carmen Orozco-Mosqueda, M., and Glick, B. R. 2016. Plant growth-promoting bacterial endophytes. Microbiol. Res. 183:92-99.

Scherwinski, K., Grosch, R., and Berg, G. 2008. Effect of bacterial antagonists on lettuce: Active biocontrol of Rhizoctonia solani and negligible, short-term effects on nontarget microorganisms. FEMS Microbiol. Ecol. 64:106-116.

Shade, A., Jacques, M. A., and Barret, M. 2017. Ecological patterns of seed microbiome diversity, transmission, and assembly. Curr. Opin. Microbiol. 37: $15-22$.

Shannon, P., Markiel, A., Ozier, O., Baliga, N. S., Wang, J. T., Ramage, D., Amin, N., Schwikowski, B., and Ideker, T. 2003. Cytoscape: A software environment for integrated models of biomolecular interaction networks. Genome Res. 13:2498-2504.

Shu, K., Liu, X. D., Xie, Q., and He, Z. H. 2016. Two faces of one seed: Hormonal regulation of dormancy and germination. Mol. Plant 9:34-45.

Truyens, S., Weyens, N., Cuypers, A., and Vangronsveld, J. 2014. Bacterial seed endophytes: genera, vertical transmission and interaction with plants. Environ. Microbiol. Rep. 7:40-50.

Upreti, R., and Thomas, P. 2015. Root-associated bacterial endophytes from Ralstonia solanacearum resistant and susceptible tomato cultivars and their pathogen antagonistic effects. Front. Microbiol. 6:255. 
Vandenkoornhuyse, P., Quaiser, A., Duhamel, M., Le Van, A., and Dufresne, A. 2015. The importance of the microbiome of the plant holobiont. New Phytol. 206:1196-1206.

Vannier, N., Mony, C., Bittebiere, A. K., Michon-Coudouel, S., Biget, M., and Vandenkoornhuyse, P. 2018. A microorganisms' journey between plant generations. Microbiome 6:79.

Weinert, N., Meincke, R., Gottwald, C., Radl, V., Dong, X., Schloter, M., Berg, G., and Smalla, K. 2010. Effects of genetically modified potatoes with increased zeaxanthin content on the abundance and diversity of rhizobacteria with in vitro antagonistic activity do not exceed natural variability among cultivars. Plant Soil 326:437-452.

Yan, Y., Kuramae, E. E., De Hollander, M., Klinkhamer, P. G. L., and Van Veen, J. A. 2017. Functional traits dominate the diversityrelated selection of bacterial communities in the rhizosphere. ISME J. 11:56-66.

Yan, Z., Reddy, M. S., and Kloepper, J. W. 2003. Survival and colonization of rhizobacteria in a tomato transplant system. Can. J. Microbiol. 49: 383-389. 